\title{
Time Scales for Period Change in Pulsating White Dwarf Stars
}

\author{
S. O. Kepler ${ }^{1}$, J. E. S. Costa ${ }^{1}$, D. E. Winget ${ }^{2}$, M. D. Reed ${ }^{3}$, S. D. \\ Kawaler $^{3}$
}

\begin{abstract}
We have used the rate of change of pulsation period for the hot (DOV) pre-white dwarf PG1159-035 and the cool (DAV) white dwarf G117-B15A to measure their evolutionary time scales. We show that, for any multiperiodic star, we must take into account the effect of all pulsations simultaneously on the times of maximum of the pulsations to get reliable measurements of periods and phases.
\end{abstract}

\section{Introduction}

We used the Whole Earth Telescope (WET) to measure the light curves of the $T_{\text {eff }}=140000 \mathrm{~K}$ nonradially pulsating pre-white dwarf (DOV) PG1159-035, and the $T_{\text {eff }}=12500 \mathrm{~K}$ pulsating white dwarf (DAV) G117-B15A, to detect a large number of pulsations simultaneously. For PG1159-035, Winget et al. (1991) detected around 120 pulsations, while for G117-B15A Kepler et al. (1991) detected only 6 pulsations.

Winget, Hansen, \& van Horn (1983) showed the rate of period change with time for the pulsating white dwarfs is due to a competition of two evolutionary factors, the cooling of the star and the contraction rate. For the DOV stars, these two rates are fast, leading to an expected $\dot{P}$ of the order of $10^{-11} \mathrm{~s} / \mathrm{s}$, while for the DAVs contraction is negligible and the cooling causes a rate of period change of the order of $10^{-15} \mathrm{~s} / \mathrm{s}$.

\section{PG1159-035}

The large period change for the DOVs lead us to measure the change of the main period itself, after taking into account the effect of the other periodicities detected in the light curve. The earlier analysis of the data (Winget et al. 1985 ) could not take into full account the other modes present. Therefore, they underestimated the true uncertainties in phase due to interference between the individual frequencies present in the light curve. Using the WET datasets from 1989 and 1993, together with all the data from 1979 to the present, the rate of period change could be measured directly from the observed periods at different

\footnotetext{
${ }^{1}$ Instituto de Física da UFRGS, 91501-900 Porto Alegre, RS - Brazil

${ }^{2}$ Department of Astronomy, University of Texas, Austin, TX 78712, USA

${ }^{3}$ Iowa State University, Ames, Iowa 50011, USA
} 
years, and is:

$$
\dot{P}=(+13.07 \pm 0.03) \times 10^{-11} \mathrm{~s} \mathrm{~s}^{-1}
$$

a few times larger than those predicted by theoretical models, increasing the urgency to measure the rate of period change for the other observed pulsation modes.

\section{G117-B15A}

For G117-B15A, the DAV star, we could only measure the effect of the period change on the time of maximum (phase) of the pulsation. Even though we have accumulated $25 \mathrm{yr}$ of observations, the detection of the period change is not progressing as expected with the square of the length of the observing period, probably due to undetected pulsation modes, perhaps rotationally split extremely low amplitude companion modes to the main pulsation. Even though these suspected modes are below our detection limit, there is a detectable amplitude variation on the 215 -s pulsation, of the order of $20 \%$. The effect of low amplitude modes on the phase could explain the apparently non-random noise in the O-C diagram seen in Kepler et al. (1991).

Our estimate of the rate of period change for the main pulsation, including all the data from 1974 to 1999 is:

$$
\dot{P}=(2.8 \pm 1.7) \times 10^{-15} \mathrm{~s} / \mathrm{s}
$$

\section{Conclusion}

The time scales for period change $\tau \equiv P / \dot{P}$ are:

$$
\begin{aligned}
\tau_{\mathrm{PG1159-035}} & =(1.25 \pm 0.05) \times 10^{5} \mathrm{yr} \\
\tau_{\mathrm{G} 117-\mathrm{B} 15 \mathrm{~A}} & =(2.4 \pm 1.5) \times 10^{9} \mathrm{yr}
\end{aligned}
$$

The main conclusion of our research is that we need to take into account all the observed pulsation modes simultaneously, to measure either the period or the phase of a pulsation accurately.

\section{References}

Kepler, S. O., Winget, D. E., Nather, R. E., et al. 1991, ApJ, 378, L45

Winget, D. E., Hansen, C. J., \& Van Horn, H. M. 1983, Nature, 303, 781

Winget, D. E., Kepler, S. O., Robinson, E. L, Nather, R. E., \& O'Donoghue, D. 1985, ApJ, 292, 606

Winget, D. E., Nather, R. E., Clemens, J. C., et al. 1991, ApJ, 378, 326 